\title{
The Effectiveness of Social Media-Enabled Patient Communities on Health Goal Attainment: An Approach of Survival Analysis
}

\author{
Jiahe Song \\ Western Michigan University \\ carrie.song@wmich.edu
}

\author{
Pei Xu \\ Auburn University \\ pzx0002@auburn.edu
}

\begin{abstract}
Health goal striving activities are a major part of the daily lives of those fighting chronic diseases. In this study, we are interested in understanding the effectiveness of social media-enabled online patient communities on the outcome of health goal striving endeavor - health goal attainment. Applying social cognitive theory, we study the antecedents of health goal attainment from the respects of social support and selfreflection in online patient communities. We apply Survival Analysis to a data set of patients' interactions and their health goal progress from a leading online patient community. Our findings show that emotional social support can increase patients' chance to achieve their goals while informational social support does not appear to be effective. In addition, health-related selfreflection increases online patients' likelihood of goal attainment, but leisure-oriented self-reflection negatively affects the possibility.
\end{abstract}

\section{Introduction}

The modern day rise in life expectancy for the world's population has coincided with an increase in chronic conditions and diseases. According to a recent report by the Milken Institute [1], the economic burden associated with five of the most common chronic diseases in the United States was \$1.5 trillion during 2008-2010. A significant portion of the cost was due to the prevalence of these chronic diseases among Americans over the past decade. To restrain the growing economic burden, the traditional cost-ineffective medical service model, in which health experts administer complex treatments to patients and guide them in follow-up self-care, has gradually shifted toward more collaborative, community and individual empowerment approaches [2].

One initiative of such approaches is communitybased goal striving support on social media platforms. Health goal attainment of chronic diseases has long been recognized as an important indicator of patients' health states. Based on a recent poll, ninety percent of the participants set goals to manage their health, and one in three of them intended to know more on how to attain their health goals [3]. With the prevalence of social media-enabled online patient communities (OPCs), an increasing number of patients, especially chronic illness sufferers, have been engaged in goal striving activities in a variety of such communities. These online platforms appear to be the appropriate venues for patients to self-manage their health conditions. Despite its prevalence, the effectiveness of OPC on patients' self-managed goal attainment of chronic diseases is still unclear.

Online goal attainment process consists of two important components, i.e., social support from online peers and patients' self-reflection [e.g., 4]. Most online communities provide functions to support these two components. For example, DailyStrength.org was one of the first patient social platforms providing patients with goal setting functions. SparkPeople.com is another community that allows patients to monitor their weightloss goals while communicating with others. Extant literature does not provide a clear view on how social support and self-reflection during the goal attainment progress contribute or impede health goal attainment. It is argued that an improved health state, if achieved in the online social setting, cannot be separated from online peers' support as well as disease selfmanagement effort $[5,6]$. Since goal attainment process is not simply equal to health state in that the former involves one's cognition and judgment, it is not appropriate to conclude that social and self facets always act as boosters for goal attainment. ${ }^{1}$ Thus, we first investigate the general benefits of social support and self-reflection on the possibility of goal attainment (RQ1). In addition, we investigate the moderating role of goal type on the relationship between social support/self-reflection and the possibility of goal attainment (RQ2).

Grounded in social cognitive theory $[7,8]$, this study considers two aspects for each of the two aforementioned antecedents (i.e., social support and

\footnotetext{
${ }^{1}$ The World Health Organization provides specific scale and valuations of health state, which can be accessed through the link: http:/www.who.int/choice/demography/health_valuations/en/
} 
self-reflection) of goal attainment. Under social support antecedent, the two sub-categories that are included for direct effects are informational support and emotional support. For self-reflection, health-related content and leisure-oriented content reported by patients during goal progress updates are examined for their explanatory effects. Since the research focus is on goal attainment (i.e., the final point of any given health goal), survival analysis is identified as the most appropriate approach for data analysis. A unique data set is collected from a leading online patient community with social functions and goal settings. This online community offers chronic disease sufferers pre-defined goal type options and progress scales, an updateable journal feature, and a social-friendly comments section under each journal. 87 patients with complete goals (censored) and 305 patients with incomplete goals (uncensored) are gathered within the data set. This survival analysis helps us study how the possibility of goal attainment changes with the included social and cognitive factors.

Our empirical analysis reveals several noticeable findings. Firstly, we find that informational support is not an influential antecedent to health goal attainment whereas emotional support is beneficial for achieving health goals. Secondly, the two aspects of patients' selfreflection during goal updates affect goal attainment oppositely. It appears that, compare with leisure content, health-related self-reflection is the helpful factor for attaining a health goal. Lastly, patients' performance on goal attainment under the received social support and reported self-reflection is influenced by their goal types.

This research contributes to the literature and practice from the following perspectives. First, this study adds new insights in health goal attainment on social media-enabled communication platforms. Second, our study contributes to the literature by revealing the effects of informational/emotional support and self-cognitive endeavor on online goal management. Third, we suggest that patients take advantage of online healthcare communities as their strategic tools for disease self-management.

\section{Literature and Hypotheses}

\subsection{Disease Self-Management and Online Health Goal Attainment}

Disease self-management is a patient-centered approach to reduce healthcare cost while improving health status [9]. Such illness management is crucial especially for chronic condition sufferers who require long-lasting care and treatments. Many initiatives are conducted for boosting patient capability to gain selfefficacy and to solve self-identified problems. Most of the past research was related to self-management educational program provided by healthcare professionals [e.g., 10]. Recent studies, however, reveal that social and behavioral factors can be important predictors to evaluate risk and worthy for patient healthcare conditions and expenditures [11]. Social media-enabled OPCs provide users with both social and behavior capabilities to manage their health via goal striving functionality. Thus, attaining health goals online is the venue carrying a novel opportunity for research on patient self-management behavior and performance.

Online health goal attainment refers to the act of achieving a self-identified, beneficial health outcome through the assistance of OPC usage. Examples of such goals are "finish current treatment" for tumor patients, "be emotionally stable" for bipolar patients, "lose weight" for high blood pressure patients, etc. Extant research notes that goal attainment indicates a patient's health status [12]. Past studies on goals are focused on the antecedent role of goals by looking at the presence or absence of them [see 13] and the distance from them [e.g., 14]. Due to the important meaning of goal attainment for patients, however, it is crucial to explore and reveal the online factors influencing the possibility of goal attainment for patient self-management.

\subsection{Social Cognitive Theory}

Social cognitive theory (SCT) values a triadic reciprocal causation in which personal (cognitive), behavioral, and environmental factors intertwine with and influence each other [7]. In his SCT work, Bandura points out that a human's self-development, adaptation, and change are embedded in a social environment and that all individuals operate within a broad network of socio-structure influences [7]. In their review of SCT, Schwarzer and Luszczynska [15] note that SCT has been applied to many health related topics (e.g., emotional disorders, mental and physical health, etc.) and has become a fundamental resource in clinical, health, and personality psychology research. Von Korff et al. [6] observed that support from others and one's self-management effort are crucial for people dealing with chronic health conditions to achieve a better state of health. Thus, grounded in social cognitive theory, we conceptualize two sets of factors - social support (environmental) and self-reflection (cognitive and behavioral) - that can potentially influence patients' possibility to complete their health goals in online social networks.

\subsection{Social Support and Goal Attainment}


Social support refers to "a coping resource from which people may draw when handling stressors" $[16$, p. 64]. In the online environment, social support can be exchanged between any individuals with social ties. Social support occurs when two or more individuals exchange resources with the aim of benefiting the recipient side [17]. Social support can be viewed as social cognitive means in health practices [18]. When the online environment is the context of a study, researchers usually conduct the study with three types of social support as follows: informational support, emotional support, and companionship [e.g., 19].

Informational Support. Informational support refers to the type of assistance that helps define, comprehend, and cope with stressful problems (i.e., health conditions in this research) [20, 21]. Online informational support contains three subcategories: advice, referrals, and teaching [19]. According to social judgment theory [22], one might expect that people put more weight on the opinions closer to their own while discounting those distant ideas [23, 24]. In regard to combating a health goal, the support receiver (i.e., the goal initiator) is likely to receive practical, healthoriented information from those who have the same health issue, if not with the exactly same goal. It can be expected that the support receiver will take such information seriously. Research also shows that conformity in opinions is important in people's decision-making process. For patients, similarly, peers' informational support is based on peers' understanding and/or experience of a given medical condition, which is likely to be helpful for the goal initiator. In addition, prior research has suggested that informational support is given to a patient to reduce the uncertainty $\mathrm{s} / \mathrm{he}$ is facing to and guide the patient's action [25] and thus should be beneficial for those intending to boost their health states [26]. Thus, we anticipate the goal attainment possibility to be positively influenced by a high volume of received informational support from online community peers. Based on these arguments, we propose the first hypothesis:

H1. Online informational support is positively related to the likelihood of goal attainment for online patient community users.

Emotional Support. Emotional support is affective and sentimental in nature and communicates love or caring [26]. It is represented by understanding/empathy, encouragement, affirmation/validation, sympathy, and caring/concern [19]. Although it does not contain any constructive advice or suggestion, emotional support can help patients reduce their negative feeling due to any issues or reaffirm their self-efficacy due to a noticeable progress. This type of social support acts like stimulant directly affecting patients' mood state and emotion. In other words, emotional support can make patients feel emotionally better. As a result, patients are more likely to keep striving on their goals with less tendency to give up if they can receive more emotional support. Research has shown that better mood state helps patients achieve a better health state [27]. Thus, this social cognitive aspect of the use of online patient communities is likely to increase users' chance to finish their health goals. Therefore, we propose the second hypothesis as follows:

H2. Online emotional support is positively related to the likelihood of goal attainment for online patient community users.

\subsection{Self-Reflection and Goal Attainment}

Self-reflection is under another broader concept called self-regulation [2] in goal-related literature. In order to improve health goal progress and eventually achieve health goals, self-regulation is recognized as a crucial cognitive process for patients. Self-regulation is defined as the "self-generated thoughts, feelings, and actions that are planned and cyclically adapted to the attainment of personal goals" [28, p. 14]. Self-regulation is represented as a feedback loop $[29,30]$ in which patients periodically and constantly compare what they are/have been experiencing to what they intend to experience. This comparing mechanism, powered by self-striving activities, helps patients moving closer toward the health goal completion point. If the endeavor is not satisfied by the person's comparator mechanism, the feedback loop may be broken and the health goal might be left incomplete. Febbraro and Clum [31] note that self-reflection, the process in which individuals compare their current behavior against their goals, enables progress. In his theoretical work on social cognitive theory, Bandura [8] also notes that monitoring performance has little meaning without the comparison mechanism in human cognitive process. For those who pursue health goals in an online community, the selfevaluated or self-reflected content generated by them is a critical asset indicating whether these online patients keep their striving endeavor moving further along.

Since self-reflection in online patient communities is exposed in social environment, it is common for patients to post both health-related content and leisure-oriented content for the purpose of interaction. Patientgenerated-content (PGC) focusing on health condition and goal striving experience can be considered as a high-concentrated reflection for health goal attainment, and PGC focusing on leisure topics, on the other hand, can be considered as a low-concentrated reflection for health goal attainment. Thus, the more high- 
concentrated reflection content posted along the goal striving journey, the more likely a patient can rise up the possibility of health goal attainment. Otherwise, the patient is likely to decrease the rate of health goal attainment. This argument supports the following hypothesis:

H3. Self-reflection expressing health-related content can help patients increase the likelihood of goal attainment (H3a), whereas self-reflection emphasizing leisure-oriented content will decrease the likelihood (H3b).

\subsection{The Moderating Role of Goal Types}

In this study, we also consider the likelihood of health goal attainment to vary per different goal types. This is because that a goal type not only reflects the nature of the associated health condition but also indicates the level of difficulty a health goal may be achieved. In other words, a better planned, more manageable health goal may be easier to attain under the influence of social and cognitive factors. For example, given two individuals who receive social support and conduct self-reflection in a community, the one with a type of mental disorder may take more time or has a lower likelihood to attain a goal called "be mentally stable" than the other one with cancer to attain a goal called "complete my current chemotherapy treatment." Thus, we present our last hypothesis as follows:

H4. The effect of social support and self-reflection on the likelihood of goal attainment varies by goal type.

\section{Methodology}

\subsection{Research Context}

Our research context is a large online patient community launched in 2006. Before our data collection, the website had more than 400,000 members participating in over 500 health support groups. All the support groups were predefined by the website rather than generating by the users. Thus, each online support group has a clear focus on a specific disease or medical concern. The prior focus of the website is on chronic illnesses (e.g., mental disorder, cancer, diabetes, etc.). The website also has support groups for severe family and relationship issues (e.g., abuse, divorce, etc.) and major life style topics (e.g., diets and weight maintenance). An individual can voluntarily choose to become a registered member of this patient community, and all the available functionality (e.g., forum, support groups, social function, goal monitor, etc.) is free of charge. After filling out the illness profile page, a patient (user) will be assigned to all the related support groups. Online patients can also choose among showing the entire personal page to the public, showing selected content to the public, or showing personal content to online friends only.

To use the goal function and track the progress, a patient should first either choose a goal type from a predefined goal list provided by the website or create one based on the health issue. The website provided the most representative goal types based on the focuses of the health support groups; thus, most of the members would select one of the predefined goals. The progress on the goal is set at $0 \%$ if there is no additional update. Afterward, the members will start monitoring and tracking their goal progress. Goal progress is preset by $5 \%$ increase per interval going upward. Also, goal progress is a self-reported value provided by each patient during every time of online goal update. Any given health goal is viewed as completed (or attained) when the patient reports $100 \%$ on the goal. Figure 1 demonstrates the goal progress diagram and the goalrelated journals, which contain self-reflection and social support contents.

\subsection{Data Collection}

For patients with chronic diseases, it usually took them a few months to two years to complete a health goal. ${ }^{2}$ Thus, we collected two years of patient goal activities since December 2007 when the website launched the health goal management function.

We chose 13 support groups (see Appendix A) to collect data for the following reasons. (1) Group members tended to read other people's goal updates rather than managing their own initiatives. Thus, we needed groups with enough members engaging in their goal management activity. (2) Since the research focus is on health goals, we did not include family and relationship related support groups (e.g., parenting, divorce, etc.).

We employed a web crawler to collect data from this patient community. The complete information about the selected groups and the associated goal types along with the goal completion rate are shown in Appendix A. We selected the most popular health goal within each support group as the focus goal. For example, "Lose

\footnotetext{
${ }^{2}$ We conducted a simply distribution analysis on goal start and end time. We found that the goal initiators usually needed up to two years on average to complete a health goal.
} 
Weight" was the most popular goal in "High Blood Pressure" and "Diets \& Weight Maintenance" groups; the goal type is also relevant to members' health in these two groups. Thus, we selected "Lose Weight" as the focus goal in these two groups. Similarly, other four types of goals were selected from the other groups. It is possible that one patient had more than one health condition and joined more than one group among these 13 groups. Thus, duplicate information was eliminated before we finalized the data set. Applying our criteria and the time window, a total of 392 online patients were included in the panel data set. 87 patients managed to complete their goals within the research window, while the rest 305 left their goals unfinished.

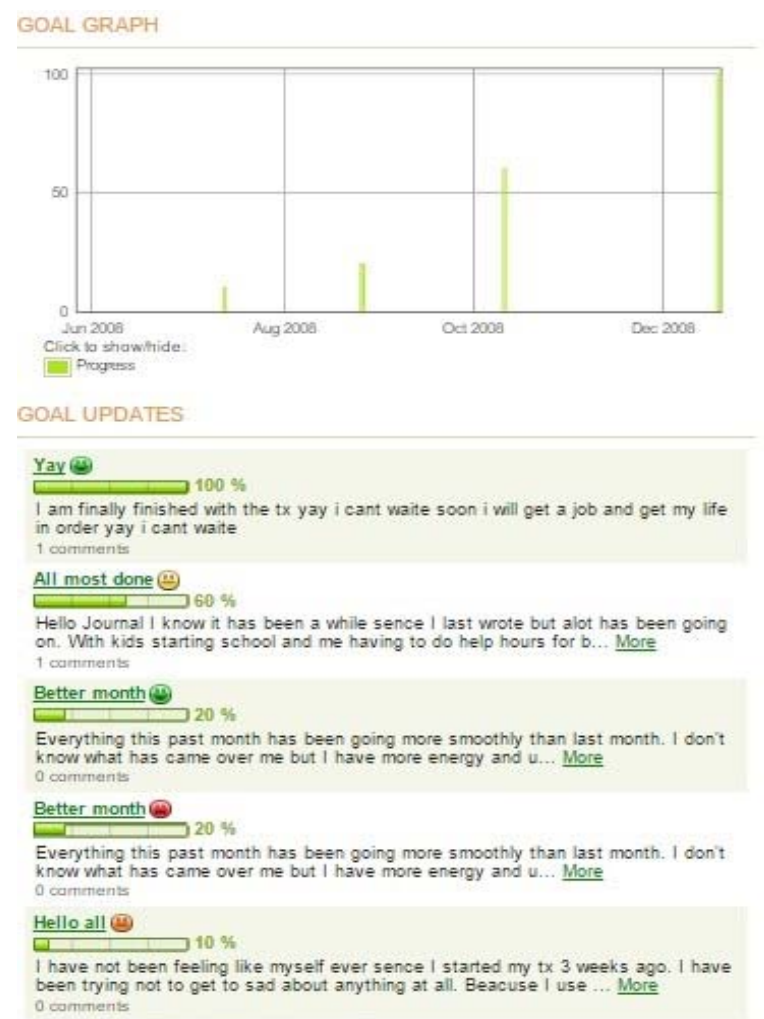

Figure 1. Goal progress diagram and goal update journals on patient goal page

\subsection{Variables}

Dependent variable. The dependent variable, $\lambda(t)$, is the hazard rate measuring the probability that a patient will experience goal attainment at time $t$. It is derived from goal duration, which represents the time spent from the goal start date to the end date. If a patient experienced health goal attainment before the end of the study period, then the end date is the actual goal end date. Otherwise, the patients are right-censored and the end date is the last day of the research window. Goal duration is assumed to have a continuous probability distribution $f(t)$. Thus, the probability that the duration will be at least $t, S(t)$, is defined as:

$$
S(t)=\operatorname{Prob}(T \geq t)=1-\operatorname{Prob}(T \leq t)=1-\int_{0}^{e} f(s) d s
$$

Since hazard rate is the probability that the duration will end after time $t$ given that it has lasted until time $t$, we define $\lambda(t)=f(t) / S(t)$.

Independent variables. (1) Informational represents the number of times online friends provide informational support to a patient. (2) Emotional represents the number of times online friends give emotional support to the patient. (3) HealthUpdate is the number of health-related content posted in patient's health goal update entries to measure health-related selfreflection. (4) LeisureUpdate is the number of leisureoriented content posted in the health goal update entries to measure the leisure-oriented self-reflection.

Control variables. (1) Tenure indicates the length in time since a patient became the member of the online community and is measured by days. (2) NumberOfUpdate shows the total number of goal updates a patient posted until the goal attainment is censored or the end of the research window. (3) HealthResponse captures the number of patient's health-related response to online peers in the comments section. (4) LeisureResponse captures the number of patient's leisure-oriented response to online peers in the comments section.

\subsection{Content Analysis}

The purpose of this content analysis is to construct the aforementioned explanatory variables, by coding text content. The items under social support are adopted from Bambina's research [19]. A complete version of Bambina's definitions and coding scheme for informational and emotional support is shown in Table 1 . We conducted a directed content analysis [32] to code social support categories from the collected online records by goal update. A total of 2570 records (goal updates) required to be coded. The coding started with a clear definition of each category. Then, two coders were involved to independently code the text, and the reliability of the content analysis was checked for confirming the results. We discuss the coding process in detail in this section.

We followed Krippendorf's [33] and Landis and Koch's [34] suggestions on categorical content analysis in this research. First, a meeting was held between two researchers (as coders) to understand and discuss the concept and definitions together. A coding book (see Appendix B) was generated during the meeting. Second, we conducted a pilot coding. 30 patients' goals, or 197 records, were randomly selected from the data set to test 
whether the pilot coding result exceeds the threshold kappa value of $0.7[33,34]$. The kappa value of 0.7 indicates that the agreed understanding between coders is significantly higher than what can be concluded by chance. The coders conducted two rounds of independent coding until they reached the threshold kappa value of 0.7 . During the third round, each coder coded other 40 patients' goals (248 records), and the result exceeded the kappa value of 0.7 . This pilot coding confirmed that the coding book is robust. Third, the two coders separately coded the rest of the data set $(2125$ records). Last, all the codes were documented in the panel data set for the data analysis stated in the next section.

Table 1. Coding scheme for informational and emotional support adopted from Bambina [19]

\begin{tabular}{ll}
\hline $\begin{array}{l}\text { Support } \\
\text { Category }\end{array}$ & Support Subcategory \\
\hline Informational & Advice \\
Support & Referral \\
& Teaching \\
\hline & Understanding/Empathy \\
Emotional & Encouragement \\
Support & Affirmation/Validation \\
& Sympathy \\
& Caring/Concern \\
\hline
\end{tabular}

\subsection{Survival Analysis}

Since the research focus is the final success on health goals (i.e., health goal attainment), survival analysis and hazard ratio is the appropriate approach. We used a semi-parametric model, Cox proportional hazard regression, to analyze the health goal attainment data as all the predictors in the model are continuous variables [35]. The model is specified as follows:

$$
\lambda\left(t \mid X_{t}\right)=\lambda_{0}(t) \exp \left(X_{t}^{t} \beta\right)
$$

In this model, $\lambda_{0}(t)$ is a baseline hazard function that describes the risk for patients with $X_{t}=0$, who serves as a reference cell. $\exp \left(\boldsymbol{X}_{t}^{i} \beta\right)$ is the relative risk, a proportionate increase or reduction in risk, associated with the set of characteristics $X_{i}$. The model for this research with all the $X_{t}$ and control variables is shown as follows:

$$
\lambda\left(t \mid X_{i}\right)=\left(\begin{array}{c}
\beta_{1} \log \text { Informational }_{i} \\
+\beta_{2} \log \text { Emotional }_{i} \\
+\beta_{3} \log \text { VerbalUpdate }_{i} \\
+\beta_{4} \log \text { LeisureUpdate }_{i} \\
+ \text { controls }
\end{array}\right),
$$

where cantrats represents all the control variables in the research.

We conducted the Schoenfeld and scaled Schoenfeld residuals plots individually to check the proportionality assumption on each independent variable. The p-value of larger than 0.05 for each term indicates that all of the terms should be kept in the model. We plotted the CoxSnell residuals to check the goodness of fit. The plot indicates that the model fits well.

We compute the statistical power for a two-sided, 0.05 level test to detect an effect size (log hazard-ratio) of 1 for each of the independent variables, given our sample size is 392. After adjusting the censoring rate and the covariance among variables, we find the statistical powers for the independent variables are all greater than $98 \%$, which is well above the suggested $80 \%$ level. Thus, we conclude that our sample size is sufficient to investigate the effect of the independent variables in a Cox proportional hazard regression.

\subsection{Results}

The Cox proportional hazard regression results are reported in Table 2. Model 1 includes only control variables. It shows that two control variables, Tenure and NumberOfUpdate, are significantly associated with the hazard rate of health goal attainment, but the effects are negative. Model 2 includes the main effects along with the control variables. The results show that all the main effects are significantly related to the dependent variable in this study except informational support.

Table 2. Estimated coefficients of Cox Proportional Hazard Model on the possibility of goal attainment

\begin{tabular}{lll}
\hline Variable & Model 1 & Model 2 \\
\hline logTenure & $\mathbf{- 1 . 8 3 4 * * *}$ & $\mathbf{- 1 . 8 5 6 * * *}$ \\
& $(0.242)$ & $(0.248)$ \\
logNumberOfUpdate & $\mathbf{- 0 . 4 6 0 *}$ & $\mathbf{- 1 . 2 6 4 * * *}$ \\
& $(0.228)$ & $(0.284)$ \\
logHealthResponse & -0.140 & -0.486 \\
& $(0.406)$ & $(0.425)$ \\
logLeisureResponse & -0.245 & -0.138 \\
& $(0.476)$ & $(0.496)$ \\
\hline logInformational & & -0.412
\end{tabular}

$(0.217)$ 


\begin{tabular}{|c|c|c|}
\hline \multicolumn{2}{|l|}{ logEmotional } & $\begin{array}{l}\mathbf{0 . 6 4 7 * * *} \\
(0.155)\end{array}$ \\
\hline \multicolumn{2}{|l|}{ logHealthUpdate } & $\begin{array}{l}\mathbf{0 . 6 5 1} * * * \\
(0.189)\end{array}$ \\
\hline \multicolumn{2}{|l|}{ logLeisureUpdate } & $\begin{array}{l}-\mathbf{0 . 3 8 5 * *} \\
(0.134)\end{array}$ \\
\hline Number of parameters & 392 & 392 \\
\hline Log-likelihood & -375.0 & -347.4 \\
\hline Chi-squared & 98.50 & 153.8 \\
\hline
\end{tabular}

In $\mathrm{H} 1$, we proposed that informational support boosts the chance of goal attainment for online patient community users. As shown in Table 2, the coefficient for logInformational is negative and not significant. Thus, we did not find support for H1. In H2, we posited that emotional support increases the possibility of goal attainment for online patient community users. As shown in the results, the coefficient of logEmotional is positive and significant (hazard ratio $=1.911, \mathrm{p}<0.001$ ), thus supporting H2. It suggests that one unit increase in logEmotional is associated with $91.1 \%$ increase in the hazard rates. In H3a-b, we hypothesized that healthrelated self-reflection content increases the possibility of goal attainment (H3a) whereas leisure-oriented selfreflection content decreases the possibility $(\mathrm{H} 3 \mathrm{~b})$. The results show that the hazard ratios for $\mathrm{H} 3 \mathrm{a}$ and $\mathrm{H} 3 \mathrm{~b}$ are $1.918(p<0.001)$ and $0.680(p<0.01)$ respectively. Thus, H3a-b are also supported.

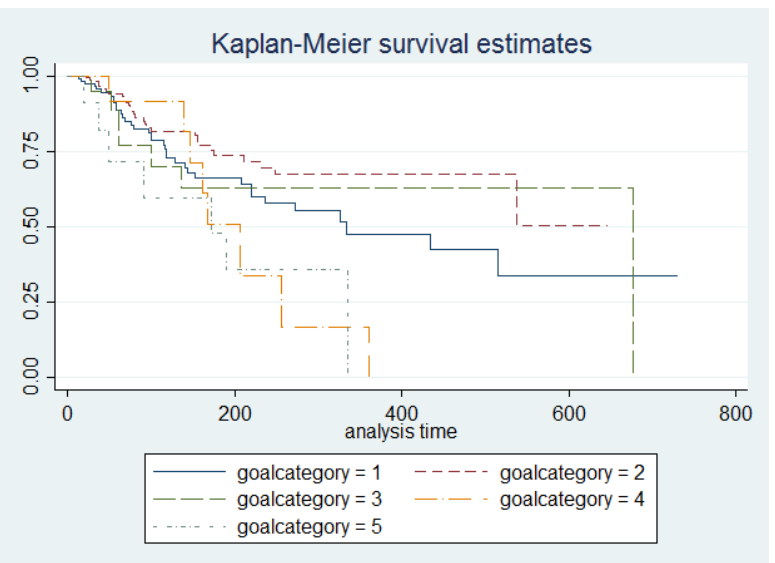

Figure2. Kaplan-Meier survival estimates by goal types (1-Improve Mental Disorder; 2-Lose Weight; 3-Manage Diabetes; 4-Beat HCV; 5Complete Cancer Treatment)

The moderating effect of goal types was proposed in H4. The survival functions by goal type show that, at any point in time, goals are associated with different survival rate (See figure 2). Test for equality of survival functions among the groups suggests the observed differences are significant $(p<0.01)$. Patients with wellplanned goals, such as Beat HCV and Complete Cancer Treatment, are more likely to attain their goals than patients with goals involving higher uncertainties. Thus, H4 is supported.

\section{Discussion and Conclusion}

Disease self-management has entered a new age when healthcare social media communities become increasingly important in patients' daily life [36, 37]. Patients are more engaged in managing their health through setting and monitoring goals with the presence of online peers and friends. Besides the convenience of interacting with peers $24 / 7$, social media-enabled health goal striving activity also challenges our knowledge on the effectiveness of the communication patterns in regard to health goal attainment. Applied the Cox proportional hazard regression model in this research, we studied the relationships between social support, self-reflection, and the possibility of goal attainment from a social cognitive theory perspective. In this section, we discuss the key findings, research contributions, practical implications, limitations and suggestions for future research.

\subsection{Findings}

This research has several key findings. First, the main effects of informational and emotional support appear to be different on the possibility of health goal attainment. If a patient does not provide any selfreflection with either health-related or leisure-oriented content during goal updates (i.e., no health or leisure update in model 2), this person's possibility to finish the goal will not be changed as the amount of the received informational support increases. However, this possibility increases with the increase of the received emotional support. Thus, practical health information from non-professional peers does not help the purpose of health goal setting (i.e., health goal attainment); rather, emotion or intuition-driven information from others increases the chance for patients' success on their health goal management.

Second, patients' self-reflective updates on healthrelated experience and leisure events also affect health goal attainment in different directions. If a patient does not receive any social support (i.e., no informational or emotional support in model 2), s/he will have a higher chance to attain the health goal by providing healthrelated self-reflection. The possibility will be decreased if the patient focuses on sharing leisure activities and daily life. 
Third, this study confirms the moderating effect of goal type on the relationship between the preceding four antecedents and the possibility of health goal attainment. The results suggest that the level of manageability of a goal is positively related to the link.

\subsection{Implications for Research and Practice}

A couple of research contributions are made from the study. First, this study adds new insights for health goal attainment on social media-enabled communication platforms. We conducted a survival study on the possibility of health goal attainment with the presence of online social support and self-reflection. The design of the study allowed us to involve both environmental (peer support) and cognitive (selfreflection) factors in patients' behavior on goals. To the best of our knowledge, this is one of the first research investigating goal attainment in the social media setting. Second, although extant studies recognize the crucial effect of social support on patients' health [e.g., 26], it is unclear whether social support is beneficial for patients' self-striving behaviors (e.g., health goal attainment). Our study contributes to the literature by revealing the different effects of social support (i.e., informational support and emotional support) on patients' goal attainment likelihood. More specifically, informational support, by itself, may not play a notable role in patients' goal attainment. Emotional support, on the other hand, is beneficial in the relationship with health goal attainment possibility. This means that positive emotion and intuition may be more crucial than informational support during health goal management behavior since the former support is likely to increase patients' confidence in reaching their goals. Additionally, this research echoes Yan et al. [38] research on patients' cognitive capabilities (e.g., the amount of information to be processed) but push it further to connect with a meaningful health outcome (i.e., goal attainment possibility), which provides building blocks for future research related to patients' health outcome enabled by online cognitive process.

In addition, the study also provides some important implications for practice. First, we suggest that online patients set their goals in a more specific manner as a well-thought, manageable objective is likely to benefit patients more from social/cognitive influencers toward goal attainment. Second, although setting health goals, especially chronic condition goals, does not always lead to the winning of a battle with a disease, being able to finish a goal has significant meaning to patients' disease management. Therefore, offline patients should be encouraged by their caregivers to go online and strive their goals with the presence of social influence.

\subsection{Limitations and Future Research}

Finally, we acknowledge the limitations of this study and point out some directions for the future research. First, other comparisons may be conducted through more grouping methods, such as female vs. male and mental vs. physical conditions, for other potential insights. Second, we collected data from the active condition groups on goal striving activities. However, the less active groups may carry their unique characteristics that were not uncovered by our data set. Since additional desirable data are truly hard to obtain, more robustness checks may be conducted to help verify the results of the survival analysis. Finally, more control variables can be included to help reduce the omitted variable bias. Examples of such variables are the number of support givers, the number of strong ties and weak ties, etc.

\section{References}

[1] A. Chatterjee, S. Kubendran, J. King, and R. DeVol, "Checkup Time: Chronic Disease and Wellness in America," Milken Institute, 2014. Retrieved from:

http://www.milkeninstitute.org/publications/view/618.

[2] S. Maes and P. Karoly, "Self-Regulation Assessment and Intervention in Physical Health and Illness: A Review," Applied Psychology, vol. 54, no. 2, pp. 267-299, 2005.

[3] Welltok, "Senior Health and Technology Survey," Welltok, Inc., 2015. Retrieved from:

http://www.prnewswire.com/news-releases/new-nationalsurvey-shows-that-seniors-desire-to-improve-health-doesntfade-with-age-300222245.html.

[4] J. Song, P. Xu, K.B. Lee, T.A. Byrd, and D. Paradice, "Social Media-Enabled Patient-to-Patient Interaction and Health Goal Striving: An Empirical Investigation," Unpublished Manuscript, 2017.

[5] A. Coulter, V.A. Entwistle, A. Eccles, S. Ryan, S. Shepperd, and R. Perera, "Personalised Care Planning for Adults with Chronic or Long-Term Health Conditions," The Cochrane Library, 2015.

[6] M. Von Korff, J. Gruman, J. Schaefer, S.J. Curry, and E.H. Wagner, "Collaborative Management of Chronic Illness," Annals of Internal Medicine, 127(12), 1997, pp. 1097-1102.

[7] A. Bandura, "Social Cognitive Theory of Mass Communication," Media Psychology, 3(3), 2001, pp. 265-299.

[8] A. Bandura, "Social Cognitive Theory of Self-Regulation," Organizational Behavior and Human Decision Processes, 50(2), 1991, pp. 248-287. 
[9] K.R. Lorig, D.S. Sobel, P.L. Ritter, D. Laurent, and M. Hobbs, "Effect of a Self-Management Program on Patients with Chronic Disease," Effective Clinical Practice: ECP, 4(6), 2000, pp. 256-262.

[10] T. Bodenheimer, K. Lorig, H. Holman, and K. Grumbach, "Patient Self-Management of Chronic Disease in Primary Care," Jama, 288(19), 2002, pp. 2469-2475.

[11] J.H. Hibbard, J. Greene, R. Sacks, V. Overton, and C.D. Parrotta, "Adding a Measure of Patient Self-Management Capability to Risk Assessment Can Improve Prediction of High Costs," Health Affairs, 35(3), 2016, pp. 489-494.

[12] D.B. Reuben and M.E. Tinetti, "Goal-Oriented Patient Care-An Alternative Health Outcomes Paradigm," New England Journal of Medicine, 366(9), 2012, pp. 777-779.

[13] E.A. Locke and G.P. Latham, "Building A Practically Useful Theory of Goal Setting and Task Motivation: A 35Year Odyssey," American Psychologist, 57(9), 2002, p. 705.

[14] P.B. Goes, C. Guo, and M. Lin, "Do Incentive Hierarchies Induce User Effort? Evidence from An Online Knowledge Exchange," Information Systems Research, 27(3), 2016, pp. 497-516.

[15] R. Schwarzer and A. Luszczynska, M. Conner and P. Norman, Eds. Social Cognitive Theory (Predicting Health Behaviour). London, England: Open University Press, 2005, pp. 127-169.

[16] P.A. Thoits, "Stress, Coping, And Social Support Processes: Where are We? What Next?," Journal of Health and Social Behavior, 1995, pp. 53-79.

[17] S.A. Shumaker and A. Brownell, "Toward A Theory of Social Support: Closing Conceptual Gaps," Journal of Social Issues, 40(4), 1984, pp. 11-36.

[18] A. Bandura, "Health Promotion by Social Cognitive Means," Health Education \& Behavior, 31(2), 2004, pp. $143-$ 164.

[19] A. Bambina, Online Social Support: The Interplay of Social Networks and Computer-Mediated Communication. Cambria Press, 2007.

[20] P.A. Thoits, "Mechanisms Linking Social Ties and Support to Physical and Mental Health," Journal of Health and Social Behavior, 52(2), 2011, pp. 145-161.

[21] S. Cohen and T.A. Wills, "Stress, Social Support, and The Buffering Hypothesis," Psychological Bulletin, 98(2), 1985, p. 310.

[22] M. Sherif and C.I. Hovland, Social Judgment: Assimilation and Contrast Effects in Communication and Attitude Change. New Haven, CT: Yale University Press, 1961.
[23] R.M. Perloff, The Dynamics of Persuasion: Communication and Attitudes in The Twenty-First Century. Routledge, 2010.

[24] I. Yaniv, "Receiving Other People's Advice: Influence and Benefit," Organizational Behavior and Human Decision Processes, 93(1), 2004, pp. 1-13.

[25] K.Y. Huang, I. Chengalur-Smith, and W. Ran, "Not Just for Support: Companionship Activities in Healthcare Virtual Support Communities," Communications of the Association for Information Systems, 34(29), 2014, pp. 561-594.

[26] L. Yan and Y. Tan, "Feeling Blue? Go Online: An Empirical Study of Social Support Among Patients," Information Systems Research, 25(4), 2014, pp. 690-709.

[27] S.D. Pressman and S. Cohen, "Does Positive Affect Influence Health?," Psychological Bulletin, 131(6), 2005, p. 925.

[28] B.J. Zimmerman, Attaining Self-Regulation: A Social Cognitive Perspective (Handbook of Self-Regulation, no. 1). San Diego, CA: Academic Press, 2000.

[29] G.A. Miller, E. Galanter, and K.H. Pribram, Plans and The Structure Of Behavior. Adams Bannister Cox, 1986.

[30] M.F. Scheier and C.S. Carver, "Optimism, Coping, and Health: Assessment and Implications of Generalized Outcome Expectancies," Health Psychology, 4(3), 1985, p. 219.

[31] G.A. Febbraro and G.A. Clum, "Meta-Analytic Investigation of The Effectiveness of Self-Regulatory Components in The Treatment of Adult Problem Behaviors," Clinical Psychology Review, 18(2), 1998, pp. 143-161.

[32] H.F. Hsieh and S.E. Shannon, "Three Approaches To Qualitative Content Analysis," Qualitative Health Research, 15(9), 2005, pp. 1277-1288.

[33] K. Krippendorff, Content Analysis: An Introduction To Its Methodology. Sage, 2004.

[34] J.R. Landis and G.G. Koch, "The Measurement of Observer Agreement for Categorical Data," Biometrics, 1977, pp. 159-174.

[35] D.R. Cox, "Regression Models and Life-Tables," In Breakthroughs in Statistics: Springer, 1992, pp. 527-541.

[36] C. Hawn, "Take Two Aspirin and Tweet Me in The Morning: How Twitter, Facebook, and Other Social Media Are Reshaping Health Care," Health Affairs, 28(2), 2009, pp. 361-368.

[37] M. Barrett, E. Oborn, and W. Orlikowski, "Creating Value in Online Communities: The Sociomaterial Configuring of Strategy, Platform, and Stakeholder Engagement," Information Systems Research, 27(4), 2016, pp. 704-723. 
[38] L. Yan, J. Peng, and Y. Tan, "Network Dynamics: How Can We Find Patients Like Us?," Information Systems Research, 26(3), 2015, pp. 496-512.

Appendix A. Detail about the Selected Goals and IIIness Groups

\begin{tabular}{cccccc}
\hline Goal Type & Support Group & $\begin{array}{c}\text { Total Group } \\
\text { Members }\end{array}$ & $\begin{array}{c}\text { Total Members } \\
\text { with The Goal }\end{array}$ & $\begin{array}{c}\text { Total } \\
\text { Completed } \\
\text { Goals }\end{array}$ & $\begin{array}{c}\text { Completion } \\
\text { Rate }\end{array}$ \\
\hline Lose Weight & High Blood Pressure & 1464 & 163 & 13 & $8 \%$ \\
& Diets \& Weight Maintenance & 12202 & 3085 & 199 & $6 \%$ \\
\hline Manage & Diabetes Type I & 1488 & 89 & 5 & $6 \%$ \\
Diabetes & Diabetes Type II & 3928 & 218 & 16 & $7 \%$ \\
\hline Beat HCV & Hepatitis C & 3693 & 122 & 39 & $32 \%$ \\
\hline \multirow{2}{*}{ Complete } & Teens with Cancer & 122 & 11 & 2 & $18 \%$ \\
Cancer & Breast Cancer & 1323 & 44 & 5 & $11 \%$ \\
Treatment & Colon Cancer & 763 & 33 & 4 & $12 \%$ \\
& Lung Cancer & 865 & 17 & 1 & $6 \%$ \\
\hline \multirow{2}{*}{ Improve Mental } & Brain/CNS Tumors & 851 & 17 & 2 & $12 \%$ \\
Disorder & Bipolar Disorder-Teen & 1506 & 92 & 9 & $10 \%$ \\
\hline 5 Goal Types & physical \& Eisorder-adult & 21615 & 1325 & 91 & $7 \%$ \\
\hline
\end{tabular}

Appendix B. Coding Book for Content Analysis

\begin{tabular}{|c|c|}
\hline $\begin{array}{c}\text { Social Support } \\
\text { Subcategory }\end{array}$ & Definition \\
\hline $\begin{array}{l}\text { Informational } \\
\text { support }\end{array}$ & $\begin{array}{l}\text { Informational support is provided to the type of assistance that helps define, comprehend, } \\
\text { and cope with stressful problems (i.e., health problems in this research). }\end{array}$ \\
\hline Advice & Giving guidance and/or making suggestions. \\
\hline Referral & Offering a source for information or assistance. \\
\hline Teaching & Passing on instructional and/or educational information. \\
\hline \multicolumn{2}{|c|}{$\begin{array}{l}\text { Examples } \\
\text { 1. I was first put on Femara. However, I had to switch to Tamoxifen as I could not tolerate the side effects of the } \\
\text { Femara... } \\
\text { 2. I suggest you look into "Zone." Eat } 3 \text { meals daily }+2 \text { snacks, just avoid bad carbs and sugar. } \\
\text { 3. It (losing weight) takes time, determination, and of course dedication, eating healthy and exercise) }\end{array}$} \\
\hline Emotional support & Emotional support is affective and sentimental in nature and communicates love or caring. \\
\hline $\begin{array}{r}\text { Understanding/empa } \\
\text { thy }\end{array}$ & $\begin{array}{l}\text { Appreciating and/or identifying with what someone is expressing often, not necessarily, } \\
\text { based on having shared a similar experience. }\end{array}$ \\
\hline Encouragement & Attempts to inspire hope and courage, to hearten. All prayers. \\
\hline & $\begin{array}{l}\text { Acknowledgement and/or positive reinforcement of someone, their ideas, attitude, or } \\
\text { actions. }\end{array}$ \\
\hline Sympathy & Expressing sorrow for or feeling troubled by another's situation. \\
\hline Caring/concern & Demonstrating regard for someone's wellobeing by being very attentive and interested. \\
\hline \multicolumn{2}{|c|}{$\begin{array}{l}\text { Examples } \\
\text { 1. I went to bed at 2:30AM and was up at } 7 \text { so I know how you feel. Isn't bipolar FUN??? LOL! } \\
\text { 2. Hugs!!! } \\
\text { 3. Gosh, that's a lot to put on your shoulders, especially when you need direction and advice. } \\
\text { 4. You've made the right choice! Just as this journey is different for each of us, so are the decisions we have to } \\
\text { make... } \\
\text { 5. I just want you to know that don't think it's you ... it's something in him ... please don't take it personally... }\end{array}$} \\
\hline
\end{tabular}

